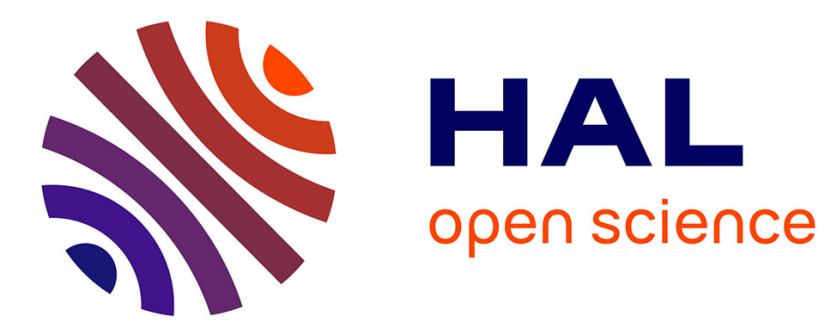

\title{
Résistivité des élastomères magnétorhéologiques
}

\author{
Nadir Kchit, Georges Bossis
}

\section{To cite this version:}

Nadir Kchit, Georges Bossis. Résistivité des élastomères magnétorhéologiques. Matériaux \& Techniques, 2009, 97 (1), pp.29-33. 10.1051/mattech/2009010 . hal-00439875

\section{HAL Id: hal-00439875 https://hal.science/hal-00439875}

Submitted on 10 Feb 2010

HAL is a multi-disciplinary open access archive for the deposit and dissemination of scientific research documents, whether they are published or not. The documents may come from teaching and research institutions in France or abroad, or from public or private research centers.
L'archive ouverte pluridisciplinaire HAL, est destinée au dépôt et à la diffusion de documents scientifiques de niveau recherche, publiés ou non, émanant des établissements d'enseignement et de recherche français ou étrangers, des laboratoires publics ou privés. 


\title{
Résistivité des élastomères magnétorhéologiques
}

\author{
N. Kchit et G. Bossis \\ L.P.M.C., UMR-CNRS 6622, Université de Nice Sophia Antipolis, Parc Valrose, \\ 06108 Nice Cedex 2, France \\ e-mail : Nadir.kchit@unice.fr; Bossis@unice.fr
}

Mots-clés :

Piézorésistivité ; composite ; percolation

\section{Key words:}

Piezoresistivity; composite; percolation

\begin{abstract}
Résumé - Des composites souples sont formés en mélangeant des particules micrométriques de nickel dans une résine réticulable à base de silicone. Le seuil de percolation dépend du traitement thermique et de la structuration de l'échantillon au cours de la réticulation. Nous présenterons les résultats relatifs à la variation de la résistance électrique de ces composites en fonction de la pression appliquée et de la température. Ces résultats seront confrontés au modèle amélioré de résistance de contact.
\end{abstract}

Abstract - Piezoresistivity of magnetorheological elastomers. Soft composites are made by mixing nickel particles of micron sizes in a curable silicon based polymer. The percolation threshold depends on the thermal treatment and on structuring the particles before curing. We present the results related to the change of the resistivity of these composites versus the applied pressure and temperature. These results are analysed with the help of a model of contact resistance.

es composites polymères conducteurs sont formés à partir de particules conductrices dispersées dans une matrice polymère isolante. Ce matériau intéresse une large communauté scientifique depuis plusieurs décennies [2]. Ses nombreux avantages (contrôle de la conductivité, flexibilité et légèreté, absorbtion de chocs mécanique), lui ont permis de trouver plusieurs formes d'applications industrielles telles que « les adhésifs conducteurs » qui remplacent les soudures au Plomb en microélectronique [3], les dispositifs de commutation, ou encore les blindages et capteurs de pressions. Beaucoup d'auteurs ont étudié la résistivité électrique des polymères chargés avec des particules conductrices en fonction de la fraction volumique $[2,4,7]$; la théorie de la percolation explique le passage de la phase isolante qui correspond à un faible taux de charge à la phase conductrice pour les taux de charge plus élevés et la mise en évidence du seuil de percolation entre les deux phases. Ce seuil dépend à la fois de la nature de la matrice polymère, de la charge et aussi de la forme et la taille des particules formant la charge.

\section{Expérience}

\section{Méthode de préparation des composites conducteurs}

Les polymères utilisés sont des élastomères RTV 141 et RTV 1062 associés à un catalyseur commercialisé par la société RHONE-POULENC. La charge est une poudre métallique de Nickel de diamètre moyen $5 \mu \mathrm{m}$. Deux type de particules ont été utilisés dans cette étude, une poudre de Nickel sans revêtement commercialisé par MERCK KgaA, et une poudre de Nickel avec revêtement d'Argent commercialisé par la société NOVAMET.

La poudre est soigneusement mélangée avec l'élastomère, d'abord à la main et ensuite dans un mélangeur pendant 1 heure pour casser le maximum $\mathrm{d}^{\prime}$ agrégats et homogénéiser le mélange. Ce dernier est dégazé sous vide pendant 15 minutes et ensuite coulé dans un moule de forme cylindrique avec deux disques en laiton de chaque côté qui servent d'électrodes lors des mesures de résistance, le mélange est mis en suite en rotation pour empêcher la sédimentation des particules pendant la 


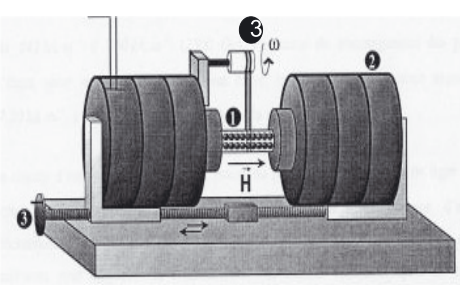

(a)

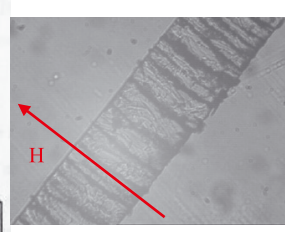

(b)
Fig. 1. (a) Dispositif de structuration des composites. 1 : échantillon, 2 : électroaimant, 3 : système de rotation. (b) Échantillon structuré à (2\% Ni-RTV141).

Fig. 1. (a) Device used for the structuration of the composite. 1: sample, 2: electromagnet, 3: rotation device; (b) sample after structuration (2\% Ni-RTV141).

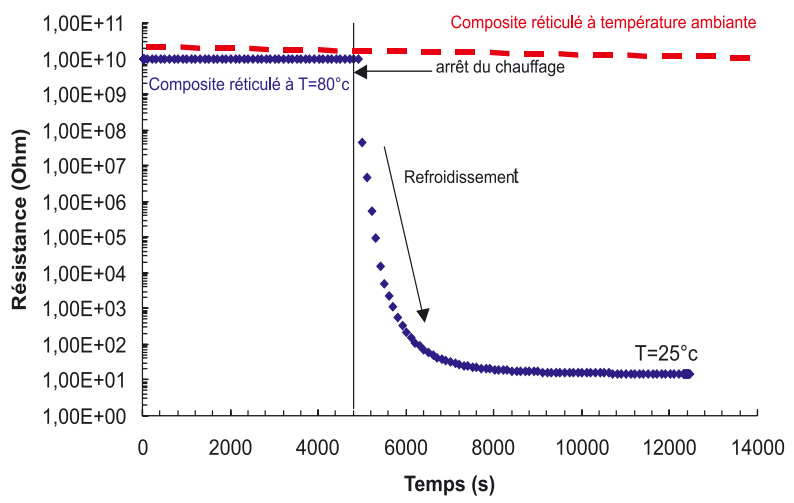

Fig. 2. Résistivité de deux composites $30 \% \mathrm{Ni}$ dans le RTV141.

Fig. 2. Resistivity of two composites $30 \%$ Ni in RTV141.

réticulation. Le temps de réticulation est très sensible à la fois à la température et à la nature des particules. À température ambiante, le mélange RTV 141 et particules de Nickel sans revêtement, réticule en $24 \mathrm{~h}$, alors qu'en chauffant à $T=80{ }^{\circ} \mathrm{C}$, sa réticulation n'est environ que 30 minutes. Le temps de réticulation à température ambiante du mélange RTV 141 et particules de Nickel couvert d'argent est environ $48 \mathrm{~h}$ et $3 \mathrm{~h}$ à $T=80^{\circ} \mathrm{C}$. Par contre, le RTV 1062 ne réticule qu'à température ambiante. Les échantillons structurés sont obtenus en appliquant un champ magnétique (Fig. 1) lors de la phase de réticulation. La dimension des échantillons est de $20 \mathrm{~mm}$ de diamètre et 2-3 mm d'épaisseur. Les mesures de piézorésistance sont effectuées sous pression uniaxiale produite par deux pistons verticaux dont l'un est monté sur un capteur de force. La résistance électrique est mesurée au moyens d'un multimètre HP.

\section{Effet de la température sur la résistivité}

On compare la résistivité finale de deux composites (RTV 141 chargé à $30 \%$ de particules

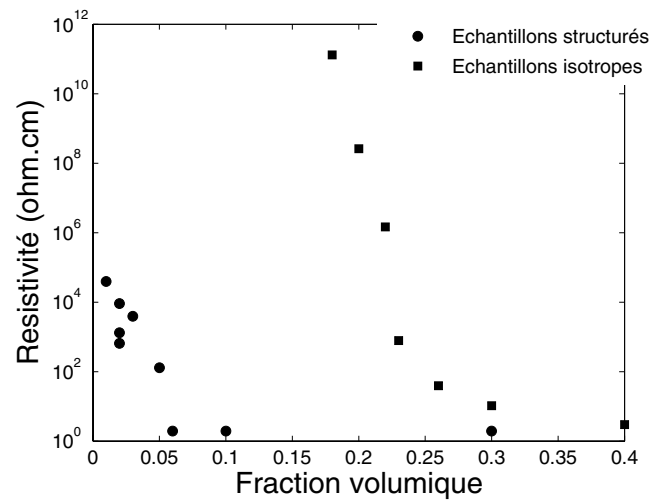

Fig. 3. Courbe de percolation pour des échantillons structurés et isotropes.

Fig. 3. Comparison of percolation between structured and isotrope samples.

de Ni). Le premier est réticulé à température ambiante (ligne discontinue), sa résistivité initiale est de quelques G $\Omega . c m$. Le deuxième échantillon est réticulé à chaud $\left(T=80^{\circ} \mathrm{C}\right)$, une fois sa polymérisation achevée, on arrête le chauffage et on enregistre la variation de la résistivité en fonction de la température de refroidissement (Fig. 2 points discontinus). La résistivité finale est de l'ordre de $100 \Omega . c m$. La chute de la résistance en fonction du refroidissement provient des contraintes internes générées par la différence de coefficient de dilatation entre le polymère et le nickel qui ressèrent le réseau de particules quand la température décroît.

\section{Effet de la structuration sur le seuil de percolation}

La figure 3 présente la résistivité en fonction de la fraction volumique de particules. Tous les échantillons à différent taux de charge isotropes et structurés ont subi le même traitement thermique (chauffage à $80^{\circ} \mathrm{C}$ puis refroidissement). Pour les échantillons isotropes, le seuil de percolation est environ $22 \%$. Par contre, les échantillons structurés sont conducteurs à partir de 5 .

\section{Piésorésistivité}

\section{Modèle de résistance de contact}

La résistivité des composites dépend de la nature du contact entre particules adjacentes. La résistance totale est la somme de la résistance de constriction et de la résistance tunnel [10].

$$
R=\frac{\rho_{\text {metal }}}{2 a}+\frac{\rho_{\text {tunnel }}}{\pi a^{2}}
$$


où $\rho_{\text {metal }}$ est la résistivité métallique des particules, a le rayon de la surface du contact et $\rho_{\text {tunnel }}$ la résistivité tunnel. Cette résistivité est calculée à partir de la densité de courant tunnel entre deux conducteur séparés par une couche isolante (Simmons [11], and Holm [12]).

La densité de courant pour des faibles tensions est donnée par la formule suivante :

$$
J=\frac{3}{8 \pi} \frac{e^{2}}{h} V \gamma \frac{\exp \left(-\gamma s_{0}\right)}{s_{0}}
$$

avec

$$
\gamma=10,24 \sqrt{\varphi} \quad\left(\mathrm{nm}^{-1}\right)
$$

$e$ la charge de l'électron, $h$ la constante de Plank, $V$ la tension en volt, $\varphi$ est la hauteur de la barrière potentiel en $\mathrm{eV}$.

- Conducteurs plans

Dans le cas de deux conducteurs plans, séparés par une couche isolante d'épaisseur $s_{0}$, la résistivité tunnel (en $\Omega$ ) est donnée par :

$$
\rho_{\text {tunnel }}\left(s_{0}, \varphi\right)=\frac{8 \pi h}{3 e^{2}} \frac{s_{0} \exp \left(s_{0} \gamma\right)}{\gamma} .
$$

- Conducteur sphérique

Dans le cas de deux conducteurs sphériques immergés dans une matrice isolante, figure 4 . La distance $g(x)$ entre les deux conducteurs n'est plus constante, mais varie en fonction de $x$. La distance la plus proche entre les deux sphères pour $x=0$ étant notée $g_{0}$ on aura :

$$
g(x)=g_{0}+\frac{x^{2}}{r} .
$$

Pour calculer la résistance électrique entre les deux sphères, on intégre la densité du courant $J$ de l'équation (2) sur la surface de la sphère, en remplaçant dans cette relation $g_{0} \operatorname{par} g(x)$.

$$
\frac{1}{R_{p}}=\frac{3}{8 \pi} \frac{e^{2}}{h} \gamma \int_{0}^{\infty} \frac{\exp (-\gamma g(x))}{g(x)} 2 \pi x d x .
$$

En remplaçant $g(x)$ par sa valeur dans l'équation (4) et pour $\gamma g_{0}>1$ l'équation (4) s'écrit:

$$
R_{p}=\frac{8}{3} \frac{h}{e^{2}} \frac{\gamma g_{0}}{r} \exp \left(\gamma g_{0}\right)
$$

\section{Particules initialement en contact}

Si les particules rentrent en contact (voir Fig. 4(2)), la résistance diminue en fonction

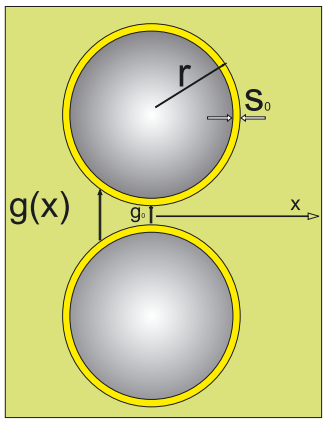

(1)

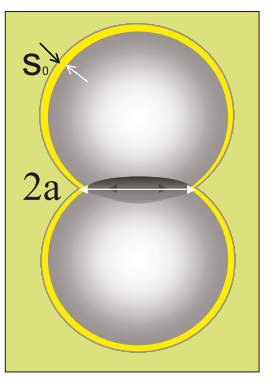

(2)
Fig. 4. Particules séparées (1) ou en contact (2). Fig. 4. Particules separated (1) or in contact (2).

de la pression appliquée à cause de l'augmentation de la surface de contact. Vu que la dureté du film d'oxyde est très grande, l'épaisseur $s_{0}$ de cette couche reste constante avec la pression, et par conséquent la résistivité tunnel (3) reste constante. Selon la nature de la déformation, soit élastique, soit plastique, la résistance de contact est donnée par:

1. Déformation élastique

$$
R_{\text {elastic }}=0,57 \rho_{\text {metal }}\left(\frac{E}{2 r F}\right)^{\frac{1}{3}}+0,26 \rho_{\text {tunnel }}\left(\frac{E}{2 r F}\right)^{\frac{2}{3}}
$$

2. Déformation plastique

$$
R_{\text {plastic }}=0,88 \rho_{\text {metal }}\left(\frac{H}{F}\right)^{\frac{1}{2}}+\rho_{\text {tunnel }} \frac{H}{F}
$$

avec $E, H$ et $R$ respectivement le module de Young, la dureté et le rayon des particules. $F$ est la force entre les particules.

Le domaine de plasticité est atteint quand la pression excède $P_{y}[13]$ :

$$
P_{y}=\frac{\pi^{2}\left(1-v^{2}\right)^{2}}{6 E} A_{y}^{3} \sigma_{y}^{3}
$$

$A_{y}$ est une constante qui dépend du coefficient de Poisson $v$ et $\sigma_{y}$ la contrainte seuil du matériau. Pour les particles de Nickel, $v=0,3 A_{y}=1,61$ et $\sigma_{y}=900 \mathrm{MPa}$, on trouve $P_{y}=0,12 \mathrm{MPa}$.

\section{Particules séparées par un film mince de polymère}

Si les particules ne sont pas en contact, (voir Fig. 4(1)), la résistance est uniquement la résistance tunnel donnée par l'équation (5). 


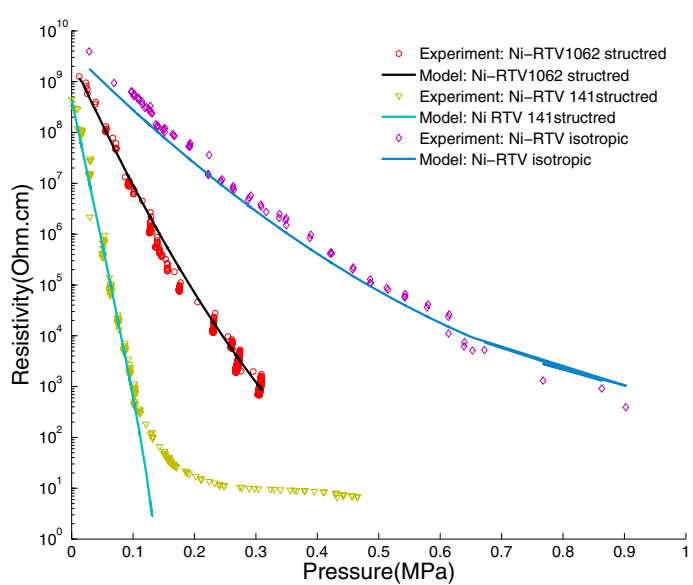

(a)

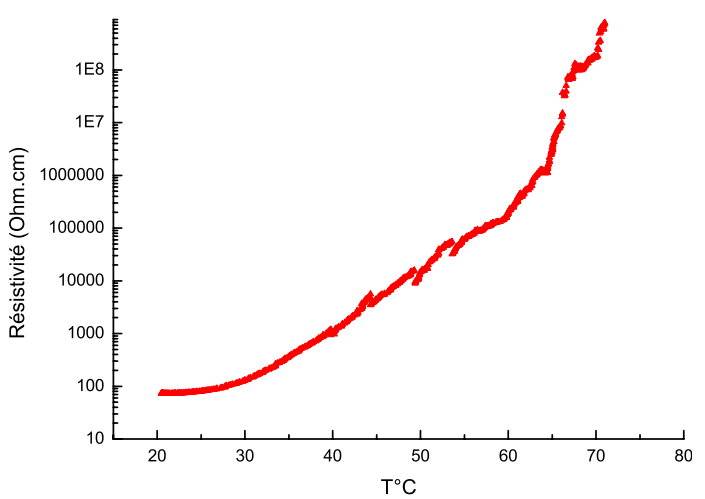

(b)

Fig. 5. (a) Les points représentent la variation expérimentales de la résistivité en fonction de la contrainte pour deux échantillons structurés à froid avec les deux élastomères RTV141 et RTV1062. Les lignes continues représentent le modèle de résistance avec : $s_{0}=2,5 \mathrm{~nm}$ et $\alpha=30$ pour le RTV1062, $s_{0}=2 \mathrm{~nm}$ et $\alpha=80$ pour le RTV141. (b) Résistivité $30 \%$ RTV $141 \mathrm{Ni}$ en fonction de la température.

Fig. 5. (a) Dots represent the experimental value of the resistivity versus the stress for two samples structured at room temperature with the two elastomers RTV141 and RYTV 1062. The solid lines represent the resistance model with $s_{0}=2.5 \mathrm{~nm}$ and $\alpha=30$ for the RTV1062 and with $s_{0}=2 \mathrm{~nm}$ and $\alpha=80$ for the RTV141. (b) Resistivity 30\% RTV141-Ni versus temperature.

Sous l'effet de la force appliquée, le gap entre particules diminue de $g_{0}$ à $g$ et la résistance change de $R_{0}$ à $R$ :

$\frac{R}{R_{0}}=\frac{g}{g_{0}} \exp \left(-\gamma\left(g_{0}-g\right)\right)=\left(1-\epsilon_{g}\right) \exp \left(-\gamma g_{0} \epsilon_{g}\right)$

avec $\epsilon_{q}$ la déformation locale entre particules adjacentes. Cette déformation est supposée proportionnelle à la déformation macroscopique :

$$
\epsilon_{g}=\alpha \epsilon
$$

où $\alpha \gg 1$ est un paramètre qui sera déterminé à partir de l'ajustement de la courbe expérimentale avec la relation :

$$
\frac{R}{R_{0}}=(1-\alpha \epsilon) \exp \left(-\gamma s_{0} \alpha \epsilon\right)
$$

\section{Effet de la matrice sur la piésorésistivité}

La figure 5a compare la piésorésistivité de deux composites structurés à température ambiante et de même fraction volumique, $30 \%$, mais avec deux matrices différentes : le RTV141 et le RTV 1062. On remarque que la résistivité du composite RTV141, en fonction de la pression appliquée, décroît plus rapidement que celle du composite RTV1062. Nous expliquons ce comportement par la différence d'énergie d'adsorption du polymère sur les particules qui se traduit par une différence sur le gap initial entre particules. À partir de la valeur initiale de la résistivité et en utilisant l'équation (5), on peut estimer le gap $g_{0}$ entre particules. Ensuite, par un fit avec l'équation (10), on obtient le paramètre $\alpha$.

Pour le RTV 141 on a $g_{0}=2 \mathrm{~nm}, \alpha=80$ et pour le RTV1062 $g_{0}=2,5 \mathrm{~nm}, \alpha=30$.

\section{Conclusion}

En polymérisant en présence d'un champ magnétique un composite chargé en particules ferromagnétiques, on obtient deux types de matériaux suivant que la polymérisation a été effectuée à chaud ou à froid. Dans le cas d'une polymérisation effectuée à chaud, l'échantillon une fois à température ambiante est conducteur et pourra servir de capteur très sensible de température puisque sa résistance décroît fortement lorsqu'on augmente la température. Au contraire, si la polymérisation a été effectuée à froid, les particules conductrices ne sont pas initialement en contact et l'application d'une pression sur l'échantillon induit une forte diminution de résistance qui peut être modélisée par une variation de l'effet tunnel avec la séparation des particules. 
Ces matériaux composites peuvent être aisément moulés, et constituer sous différentes formes, soit des capteurs de température soit des capteurs de pression facilement intégrables dans toute sorte de dispositif.

\section{Références}

[1] S.K. Bhattacharya, Metal Filled Polymers, New York: Marcel-Dekker, 1992

[2] S. Yoshikawa, T. Ota, R. Newnham, J. Am. Ceram. Soc. 73 (1990) 263

[3] J.A. Fulton, R.C. Moore, W.R. Lambert, J.J. Mottine, Proc. 39th Electronic Components Conf. IEEE, Vol. 39, 1989, 71

[4] B. Lundberg, B. Sundqvist, J. Appl. Phys. 60 (1986) 1074
[5] A. Celzard, E. McRae, J.F. Marêché, G. Furdin, J. Appl. Phys. 83 (1998) 1410

[6] X.W. Zhang, Y. Pan, Q. Zheng, X.S. Yi, Polym. Int. 50 (2001) 229

[7] F. Carmona, R. Canet, Delhaes, J. Appl. Phys. 61 (1987) 2550

[8] M. Jolly, J. Carlson, B. Munoz, Smart Mater. Struct. 5 (1996) 607

[9] G. Bossis, E. Coquelle, P. Kuzhir, Annales de Chimie - Science des Matériaux 6 (2004) 29

[10] R. Holm, Electric Contacts, Berlin: Springer, 1967

[11] J.G. Simmons, J. Appl. Phys. 34 (1963) 1793

[12] R. Holm, J. Appl. Phys. 22 (1950) 569

[13] L. Vu-Quoc, X. Zhang, L. Lesburg, J. Appl. Mech. 67 (2000) 363

[14] J. Sandlera, Polymer 40 (1999) 5967-5971

[15] N. Kchit, G. Bossis, Proc. Dijon Matériaux 13-17 nov. 2006, Dijon 